Es liegt auf der Hand, dass für eine bedarfsgerechte Steuerung des Gesundheitssystems umfassende und aktuelle Daten zur Verfügung stehen müssen. In diesem Zusammenhang benötigen wir auch unsere eigenen Daten, damit wir uns aktiv an dieser Steuerung beteiligen können! Doch die Ärztestatistik ist in die Jahre gekommen und entspricht nicht mehr den Bedürfnissen - insbesondere die reale Praxis und die heutige Arbeitsweise der Ärztinnen und Ärzte kommen durch diese Statistik nicht mehr richtig zum Ausdruck.
Deshalb machte sich die Abteilung «Daten, Demographie und Qualität» des FMH-Generalsekretariats unter der Leitung der beiden Verfasserinnen des Editorials dieser Ausgabe an eine tiefgreifende Revision der Ärztestatistik. Nach der Lektüre des Editorials werden Sie besser verstehen, worum es bei dieser Revision geht, und die Bedeutung der geleisteten Arbeit entsprechend schätzen - ein herzliches Dankeschön an die beiden Projektleiterinnen!

Jacques de Haller, Präsident der FMH

\section{Nur wer die Daten kennt, kann überzeugend planen}

Die Frage, wie viele Ärztinnen und Ärzte die Schweiz heute und zukünftig braucht, bleibt weiterhin aktuell - unabhängig vom Entscheid des Parlaments über den Zulassungsstopp. Eine bedarfsgerechte Steuerung der Leistungserbringer ist ebenfalls weiterhin ein Thema. Überzeugend ist eine Zulassungspraxis von Ärztinnen und Ärzten aus Sicht der FMH dann, wenn folgende Kriterien erfüllt sind: Die kantonalen und regionalen Versorgungs- und Bedarfssituationen werden berücksichtigt, die ambulanten, stationären und teilstationären Leistungsangebote einbezogen, und die Zulassung der Leistungserbringer durch die Kantone geschieht immer unter Einbezug der relevanten Organe der Ärzteschaft.

Die Grundlage für eine überzeugende und bedarfsgerechte Steuerung bildet in jedem Fall eine umfassende und verlässliche Datenbasis. Die FMH verfolgt und analysiert seit langem die Entwicklung der in der Schweiz berufstätigen Ärztinnen und Ärzte - die Angaben sind in der FMH-Ärztestatistik aufbereitet und auf www.fmh.ch publiziert.

In der Ärztedemographie sind in der letzten Zeit zunehmend Faktoren wichtig geworden, die in der bisherigen Ärztestatistik nicht oder zu wenig differenziert ab- gebildet werden, so zum Beispiel die Erfassung von Teilzeitarbeit. Um aktuelle und zukünftige Fragestellungen angehen zu können, revidiert die FMH aktuell ihre Ärztestatistik und ermöglicht somit ungefähr ab Ende Jahr neben den bisherigen Angaben zur Soziodemographie und zur Qualifikation (Aus-, Weiter- und Fortbildung) zusätzliche Erkenntnisse über die Ärzteschaft in der Schweiz zur ärztlichen Tätigkeit. Details entnehmen Sie dem Bericht über die Revision FMH-Ärztestatistik in dieser Nummer ab der folgenden Seite.

Mit der Ausweitung der bisherigen Ärztestatistik leistet die FMH einen zentralen Beitrag zu einer fundierten Diskussions- und Argumentationsgrundlage für die bedarfsgerechte Steuerung der Ärzteschaft. Mittelfristig braucht es ein Monitoring sämtlicher Leistungserbringer über alle Sektoren hinweg, das verlässliche Datengrundlagen für die Steuerung bereitstellt - die FMH-Ärztestatistik könnte ein Teil davon werden!

Für die Projektleitung Revision FMH-Ärztestatistik:

Martina Hersperger, Abteilungsleiterin DDQ

Esther Kraft, wissenschaftliche Mitarbeiterin DDQ 